\title{
COEXISTENCE OF SYPHILIS AND PSORIASIS
}

\section{Senthil Kumar' , K. P. Saradha², V. Anandan³, M. Manisurya Kumar4, A. S. Kalpana ${ }^{5}$}

${ }^{1}$ Senior Assistant Professor, Department of Dermatology, Stanley Medical College, Chennai. ${ }^{2}$ Senior Assistant Professor, Department of Dermatology, Stanley Medical College, Chennai.

3 Professor, Department of Dermatology, Stanley Medical College, Chennai.

${ }^{4}$ Assistant Professor, Department of Dermatology, Stanley Medical College, Chennai.

5Junior Resident, Department of Dermatology, Stanley Medical College, Chennai.

\section{ABSTRACT}

Psoriasis is a chronic inflammatory skin disease with various triggering factors. Here we report a case of psoriasis coexisting with secondary syphilis which could have been a possible trigger for exacerbation of psoriasis.

\section{KEYWORDS}

Psoriasis, Secondary Syphilis.

HOW TO CITE THIS ARTICLE: Kumar VS, Saradha KP, Anandan V, et al. Coexistence of syphilis and psoriasis. J. Evolution Med. Dent. Sci. 2016;5(46):2944-2945, DOI: 10.14260/jemds/2016/685

\section{INTRODUCTION \\ Psoriasis is a chronic inflammatory skin disease with well- defined, erythematous, scaly, indurated plaques particularly over extensor surface and scalp. Genetic susceptibility with environmental factors like trauma, infection, drugs, sunlight, smoking, alcohol, HIV, etc. are implicated in initiation and exacerbation of the disease. Syphilis is an infectious disease with florid cutaneous manifestations and mimicking various dermatoses posing diagnostic difficulties. Secondary syphilis may manifest with scaly, crusted plaques mimicking psoriasis referred to as psoriasiform syphilide.}

\section{CASE REPORT}

A 38-year-old patient, mason by occupation with 3-year history of psoriasis, on treatment, came with exacerbation of lesions for past 1 month. On examination, patient had multiple well defined scaly erythematous plaques varying in size from $1 \times 1 \mathrm{~cm}$ to $12 \times 10 \mathrm{~cm}$ involving upper limbs, chest, back, scalp and lower limbs [Figure 1]. Auspitz sign was positive. Scaly hyperpigmented plaques were present on bilateral palms [Figure 2] and soles. Nails showed coarse pitting with subungual hyperkeratosis. On further history taking patient had h/o painless ulcer in the glans penis 4 months ago, which healed without scarring. No treatment was taken for the ulcer. Considering the history of painless ulcer over the glans penis and exacerbation of skin lesions, psoriasiform syphilis was suspected and serological tests for syphilis and repeat biopsy done. RPR was positive in 1:64 dilution with TPHA positive in 1:128 dilution. Histopathology of skin lesion revealed focal acanthosis, hyperkeratosis and parakeratosis with Munro's microabscesses, hypogranulosis, suprapapillary thinning with focal elongation of rete pegs with sparse perivascular lymphocytic infiltrate which was consistent with psoriasis vulgaris [Figure 3]. Based on clinical and histopathological

Financial or Other, Competing Interest: None.

Submission 16-02-2016, Peer Review 18-05-2016,

Acceptance 24-05-2016, Published 09-06-2016.

Corresponding Author:

Dr. V. Senthil Kumar,

No. 3, Third Floor, Srinivas Apartment,

No. 10, Sankarapuram First Street,

Choolaimedu, Chennai-600094.

E-mail: vskumarveerappan@yahoo.in

DOI: $10.14260 /$ jemds $/ 2016 / 685$ findings, diagnosis of psoriasis vulgaris with coexisting secondary syphilis was made. Patient was given a single dose intramuscular injection of benzathine penicillin $2.4 \mathrm{MU}$, and a significant fall in titre was noted after 6 months of follow-up.

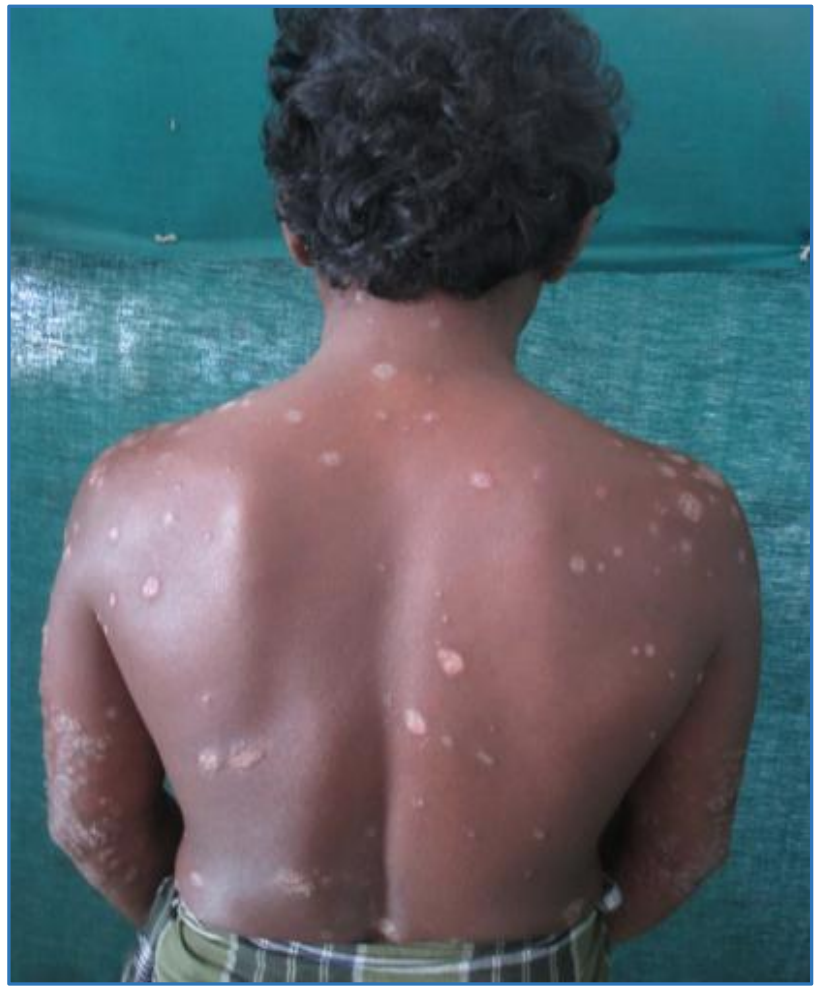

Fig. 1: Erythematous Scaly Plaques Over Back
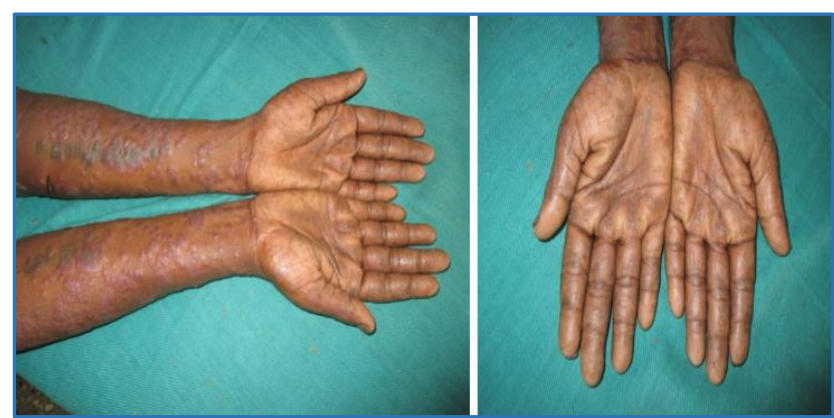

Fig. 2: Scaly Hyperpigmented Plaques, Bilateral Palms 


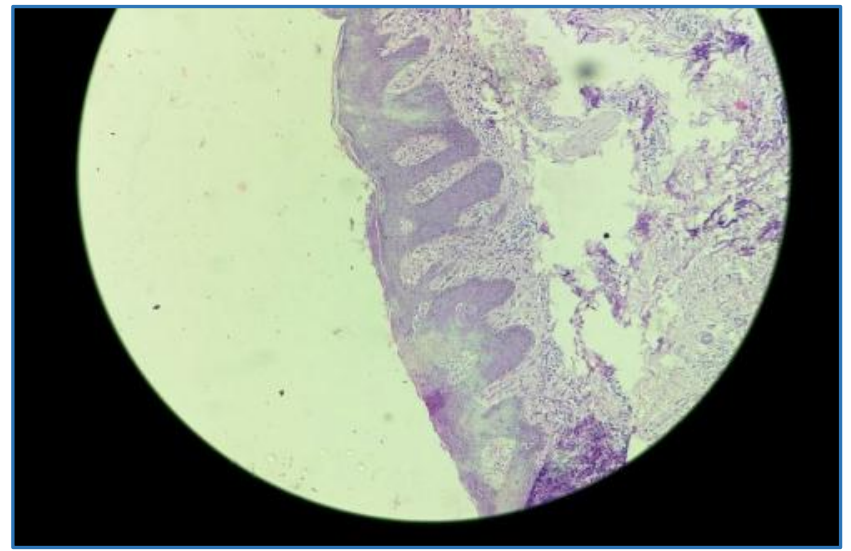

Fig. 3a: HPE showing Parakeratosis, Acanthosis, Elongation of Rete Ridges and Lymphocytic Infiltrate in Dermis

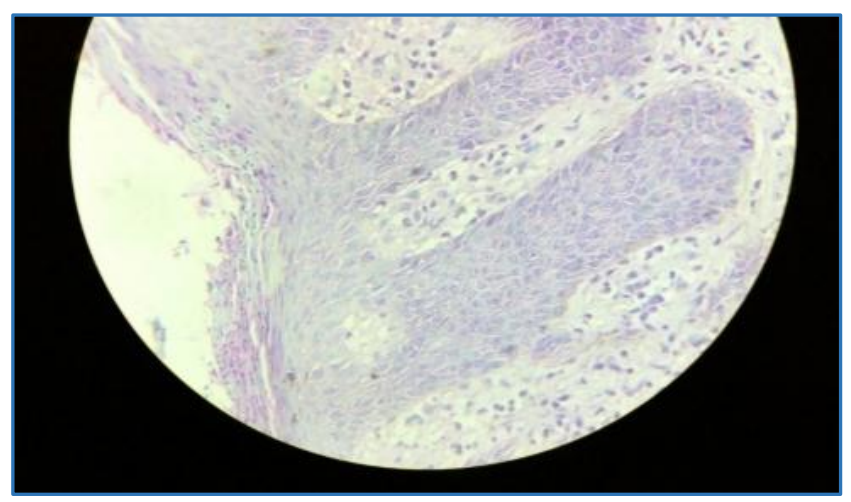

Fig. $3 b$

\section{DISCUSSION}

Psoriasis is an immunologically mediated, chronic inflammatory and proliferative condition of skin. Morphological variants of psoriasis include plaque type, guttate psoriasis, rupioid, ostraceous, elephantine psoriasis, pustular psoriasis and erythrodermic psoriasis.

There is evidence that streptococcal infection can trigger guttate and plaque type psoriasis.[1] HIV infection has been associated with psoriasis. Exacerbation of psoriasis has also been linked with skin and/or gut colonisation by Staphylococcus aureus, Malassezia and Candida albicans.[2] Possibility of primary HPV infection triggering psoriasis has also been reported.[3]

There is also a case report of secondary syphilis on a psoriatic patient, presenting with psoriatic flare and infiltrated atypical lesions which was consistent histologically with secondary syphilis. ${ }^{[4]}$
A case of secondary syphilis mimicking palmoplantar psoriasis has been reported in an HIV patient.[5] In our case, patient had history of unprotected sexual contact with multiple sex workers and history of painless ulcer over glans penis that healed without treatment. Psoriasiform syphilis was suspected, but the biopsy results were consistent with that of psoriasis vulgaris. The psoriatic flare up could be induced by the syphilitic infection. Though the patient had high titre positivity for VDRL and TPHA, histopathology did not reveal the presence of plasma cells or features of endarteritis obliterans. The patient was yet to manifest any cutaneous or systemic manifestations of secondary syphilis and was diagnosed at an early stage of syphilis only due to high degree of suspicion.

Syphilis being a great imitator of diseases is to be considered in all atypical cutaneous presentations, so as to facilitate early diagnosis. Widespread screening with VDRL in all treatment resistant and atypical cases should be done. Syphilis being highly infectious in early stage, early diagnosis and prompt treatment can terminate the chain of spread. There is no case report of isolated secondary syphilis coexisting with psoriasis. This case is reported for rarity in our institution.

\section{CONCLUSION}

This case highlights the importance of screening for syphilis in all psoriatic patients with acute flare up and those resistant to treatment. Syphilitic infection may trigger psoriasis or may present as psoriasiform syphilis.

\section{REFERENCES}

1. Telfer NR, Chalmers RJ, Whale $K$, et al. The role of streptococcal infection in the initiation of guttate psoriasis. Arch Dermatol 1992;128(1):39-42.

2. Fry L, Baker BS. Triggering psoriasis: the role of infections and medications. Clin Dermatol 2007;25(6):606-15.

3. Jain SP, Gulhane S, Pandey N, et al. Human papilloma virus infection and psoriasis: did human papilloma virus infection trigger psoriasis. Indian J Sex Transm Dis 2015;36(2):201-3.

4. Pinto-Almeida T, Rosmaninho A, Sanches M, et al. Secondary syphilis on a psoriatic patient under cyclosporine: a challenging case. IJOD 2014;53(1):e35-8.

5. Bittencourt MJS, Brito AC, Nascimento BAM, et al. A case of secondary syphilis mimicking palmoplantar psoriasis in HIV infected patient. An Bras Dermatol 2015;90(3 Suppl 1):S216-9. 